\title{
The effect of different finishing and polishing procedures on the surface gloss of Bulk-Fill resin composites
}

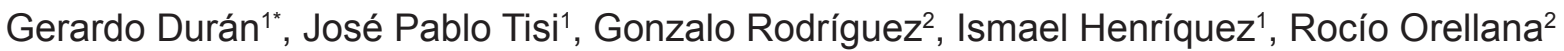 \\ 1. Assistant Professor, Facultad de Ciencias de la \\ Salud, Universidad Arturo Prat, Iquique, Chile. \\ 2. Associate Professor, Facultad de Odontología, \\ Universidad de Chile. Chile. \\ * Corresponding author: Gerardo Durán. | Address: \\ Facultad de Ciencias de la Salud, Universidad \\ Arturo Prat, Iquique, Chile. | E-mail: Gerardo. \\ duran@unap.cl \\ Trabajo recibido el 16/09/2019. \\ Aprobado para su publicación el 31/12/2019

\begin{abstract}
Statement of the problem: The literature still lacks evidence regarding which polishing techniques have the ideal clinical outcomes for bulk-fill resin composites. Purpose: This study evaluated the surface gloss of two commercially available bulk-fill resin composites after different polishing procedures and characterized their micromorphology via scanning electron microscopy. Material and Methods: 80 bulk-fill compactable composite resin discs were created. The control group was left untreated, and remaining samples were subjected to different polishing techniques. Gloss units were measured and surface morphology of disc samples was assessed. The Tukey post-hoc test was used to identify any differences. Results: Statistically significant differences were found between the different polishing systems applied to Filtek BulkFill Posterior and Tetric N Bulk-Fill. No differences were found when the same polishing system was applied for both resins. The highest gloss values were obtained in the control group and the ENA Shiny system; the lowest were obtained with SofLex XT and Soflex Spiral Wheels for the bulk-fill composite resins studied. Conclusions. Diamond pastes have the highest gloss behavior, followed by diamond rubber points. The systems with aluminum oxide discs present the lower gloss behavior. SEM images provided useful evidence, and future studies should include an evaluation over time.

KEY WORDS

Bulk-fill; Gloss; Polishing; Bulk-fill composites.
\end{abstract}

Int. J. Inter. Dent Vol. 13(1); 13-16, 2020.

\section{INTRODUCTION}

Resin-based restorative materials have improved to such an extent that they can now be considered reliable, and are appropriate for use in posterior teeth with acceptable long-term longevity ${ }^{(1)}$.

Conventional composite resins normally achieve their hardening state through a polymerization process, in which monomers such as BisGMA, UDMA and TEGDMA, among others, are able to form a large three-dimensional network or polymer chain ${ }^{(2)}$. During this process, and as a result of it, a dimensional change occurs with volumetric reduction- a phenomenon known as polymerization shrinkage, which commonly varies between $1-6 \%{ }^{(3)}$. The most commonly associated problems produced by this volumetric change may include microleakage ${ }^{(4)}$, which is a resultant of the possible adhesive or cohesive failures of the bonding interphase during polymerization that leads to the formation of secondary caries ${ }^{(5)}$. Cuspal deflection is another associated issue, which involves micro movements of the cuspids associated to a bonded layer of resin material, and corresponds to the formation of cuspal fractures and microcracks ${ }^{(6)}$.

Given the problems associated with the use of conventional composite resins in the different restorative techniques for posterior teeth, the focus of various dental companies during recent years has been the development of monomers that are capable of dissipating the stress generated during polymerization shrinkage, as well as incorporating inorganic filler particles with optical translucency properties superior to conventional composites, with the ability to transmit light and achieve acceptable conversion rates at depths close to $4-5 \mathrm{~mm}$. This group of resins are called bulk-fill resin composites ${ }^{(7)}$.

The possibility of incorporating a greater thickness of a composite resin into a tooth cavity preparation means that composite bulk-fill resins have become a more attractive focus than conventional composite resins for the restoration of posterior teeth, which is mainly due to the crucial reduction of restorative clinical time, as well as the reduction of bubbles or impurities between each composite resin layer as in the case of posterior restorations performed with stratification composite resins, which have shown a considerable increase in restorations of posterior teeth through bulk-fill compactable composite resins ${ }^{(8)}$. This has allowed more research and development in the field, including studies oriented towards the in vitro evaluation of the biomechanical behavior, as well as the phenomenon of polymerization shrinkage stress, depth of cure and degree of conversion.

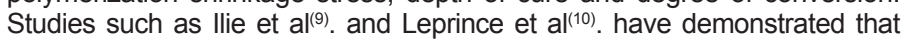

compactable bulk-fill composite resins have lower physical-mechanical properties than conventional composite resins, which is related to their flexural strength, the monomer's degree of conversion and elastic modulus. Other studies to the contrary have shown a comparable degree of conversion results with conventional composite resins ${ }^{(11)}$. When it comes to depth of cure, most of the compactable bulk-fill resins obtained an acceptable degree of conversion at $4 \mathrm{~mm}$ with extended light polymerization time and energy density; however, the degree of conversion is still lower than conventional composites $^{(12)}$.

Although a number of physical-mechanical tests have demonstrated that compactable bulk-fill resins have a lower performance than conventional composite resins in in vitro studies, clinical studies with short-term longevity and clinical performance analysis compared to conventional composite resins conclude that compactable bulk-fill resins are materials that can be safely used in clinical situations, demonstrating promising results that must be evaluated in the long term ${ }^{(13)}$.

Studies have also analyzed the optical properties of bulk-fill composites. Both flowable and high viscosity bulk-fill composite resins have superior translucency and light transmittance for blue light than conventional composite resins $^{(14)}$. When it comes to color, bulk-fill resins have less stability than conventional composite resins, even more so when they are subjected to drinks like coffee or are increased in thickness ${ }^{(15)}$. The discoloration of this type of resin is affected by the polishing procedure and the type of liquid solution that came into contact with the bulk-fill resins ${ }^{(16)}$.

According to Lefever et al. ${ }^{(17)}$, conventional composite resins have acceptable biomechanical properties which make them suitable for the restoration of teeth; however, many failures are currently associated with low levels of maintenance of surface properties like gloss and roughness. Since there is a lack of research regarding the optical behavior related to different surface treatments associated with finishing and polishing procedures of bulk-fill composites, the objective of this article is to evaluate the in vitro surface gloss of high viscosity bulk-fill resins subjected to different finishing and polishing materials.

The null Hypotheses of this study were as follows:

1. There are no significant differences in gloss values between the different polishing systems for the bulk-fill composite resin tested.

2. There are no significant differences in gloss values between resins for the same polishing system. 


\section{MATERIAL AND METHODS}

Two bulk-fill compactable composites were selected for the study: Tetric $\mathrm{N}$ Bulk Fill (Ivoclar Vivadent, Liechtenstein, Germany) color IVB, and Filtek Bulk Fill Posterior A2 (3M ESPE, St. Paul, MN).

\section{Specimen Elaboration}

80 bulk-fill composite resin discs were elaborated $(10 \mathrm{~mm}$ diameter and $2 \mathrm{~mm}$ thickness). Each sample was created by placing a single increment of resin into a stainless steel mould. Mylar strips were positioned under and over the mould, and the excess of resin was eliminated by compression between two glass plates using finger pressure. All samples were polymerized through the glass plate using a LED curing unit (Elipar Deepcure-L, 3M ESPE) calibrated at $1.470 \mathrm{~mW} / \mathrm{cm}^{2}$ according to the manufacturer's instructions (Tetric N Bulk Fill: 10 seconds, and Filtek Bulk Fill Posterior: 20 seconds). The specimens were then stored in distilled water at $37^{\circ} \mathrm{C}$ for 24 hours.

\section{Polishing Procedures}

The first group of samples was left untreated, which constituted the control group (G1). Then, the remaining specimens were roughened with 320 grit sandpaper in order to simulate the finishing technique procedure with diamond burs. A single operator performed every step of the finishing and polishing procedures. Soft pressure of $40 \mathrm{grs}$ according Antonson et al. ${ }^{(18)}$ was used by the operator (calibrated every 10 samples), using a slow speed handpiece for every procedure (WE-99 LED G, AM-25R, W\&H Dentalwerk Bürmoos/Austria $\mathrm{GmbH}$ ) at $9000 \mathrm{rpm}$, always perpendicular to the disc surface. The following polishing procedures were performed as follows:

- Group 1 (G1): Mylar strip, no finishing and polishing procedure.

- Group 2 (G2): Sof-Lex XT: Coarse, medium, fine and superfine grit discs were applied consecutively for 20 seconds. Between each grit, the discs were cleaned with an air-water spray for 10 seconds.

- Group 3 (G3): Composite Politur: An initial finishing carbide bur was applied to the entire surface (H48LQ.314.012). A pre polishing diamond rubber followed by a polishing rubber were applied for 20 seconds each. After the carbide bur and between the diamond rubbers, the discs were cleaned with an air-water spray for 10 seconds.

- Group 4 (G4): SofLex Spiral: Coarse and medium grit discs from SofLex XT system were first applied for 20 seconds each. Then, the first finishing wheel followed by the polishing and gloss wheel were applied for 20 seconds each. Between every grit disc and wheel, the discs were cleaned with an airwater spray for 10 seconds.

- Group 5 (G5): ENA Shiny: Finishing diamond rubber was initially applied for 20 seconds. Shiny A diamond paste was applied with a goat hair brush for 20 seconds. Then, Shiny B was applied with a different goat hair brush for 20 seconds, and finally, Shiny $C$ aluminum-oxide paste was used with a felt wheel for 20 seconds. Between each step, the discs were cleaned with an air-water spray for 10 seconds.

\section{Gloss measurement}

Gloss was determined by a gloss meter (Skin-Glossymeter GL 200, Courage + Khazaka Electronic $\mathrm{GmbH}$ with Cutometer dual MPA 580, Courage + Khazaka Electronic $\mathrm{GmbH}$ ) calibrated on a white surface provided by the manufacturer. The measurement area was $5 \times 2.5 \mathrm{~mm}$ with 60 degrees of angulation. Each sample was centrally placed inside a black plastic mould during the measurement in order to eliminate the influence of external and environmental light, and maintained in the same position in every measurement. Three measurements were performed in each specimen. Gloss units (GU) were calculated as the mean of the 3 measurements of each sample.

\section{Scanning electron microscopy}

Specimens were metallized with gold in sputtering equipment (Desk V, Denton Vacuum LLC, NJ, EEUU). The samples were then observed by scanning electron microscopy (SEM) at 40X, 200X, 1000X and 3500X (Jeol JSM-IT300 LV, USA Inc.) and processed by the manufacturer's software (JSM IT300 version 1.070).

\section{Statistical analysis}

With the measurements obtained from gloss (GU units), the data base was created and statistically processed using Microsoft Exce ${ }^{\bullet}$ and SPSS Statistics ${ }^{\circledast}$ v.23.0 (IBM ${ }^{\oplus}$, USA). Homogeneity of variance and normal distribution of the samples were verified by Levene and Shapiro-Wilk tests, respectively. The mean and standard deviation of gloss were calculated, obtaining graphs to make an initial observation of the data. A two-way ANOVA model was designed in order to determine differences over means between the different levels of factors, thus determine if the type of bulk-fill composite resin and the type of polisher have an effect over gloss and to determine which combination has better in vitro performance, followed by a stratified ANOVA and Tukey post-hoc test $(\alpha=.05)$.

\section{RESULTS}

Mean values and standard deviation of gloss measurement for the two bulk-fill composite resins under different polishing techniques are shown in Table 1.

Table 1:

\begin{tabular}{l|c|c|c}
\hline & $\begin{array}{c}\text { Filtek Bulk } \\
\text { Fill Posterior }\end{array}$ & $\begin{array}{c}\text { Tetric N } \\
\text { Bulk-Fill }\end{array}$ & $\begin{array}{c}\text { Mean for } \\
\text { both resins }\end{array}$ \\
\hline $\begin{array}{l}\text { G1: Mylar strips, } \\
\text { Control Group }\end{array}$ & $82.5(9.8)$ & $79.0(4.9)$ & $80.7(7.7)$ \\
\hline G2: SofLex XT & $58.3(7.2)$ & $57.2(4.5)$ & $57.8(5.8)$ \\
\hline $\begin{array}{l}\text { G3: Composite } \\
\text { Politur }\end{array}$ & $74.7(7.4)$ & $75.0(6.4)$ & $74.8(6.7)$ \\
\hline G4: Soflex Spiral & $53.3(7.1)$ & $63.4(7.3)$ & $58.4(8.7)$ \\
\hline G5: ENA Shiny & $83.6(4.8)$ & $75.5(2.9)$ & $79.6(5.7)$ \\
\hline Mean & $70.5(14.4)$ & $70.0(9.8)$ & - \\
\hline
\end{tabular}

According to the ANOVA test, there were significant statistical differences over mean values between the different polishing systems ( $p$ value $=0.000)$, but no differences were found for both bulk-fill composite resins polished by the same system ( $p$ value $=0.73$ ) (Table 2 ). The Tukey post-hoc test determined the differences, which can be seen in Table 3 .

Table 2:

\begin{tabular}{l|c|c|c|c|c}
\multicolumn{1}{c}{ Dependent Variable: GLOSS } & $\begin{array}{c}\text { Type III sum } \\
\text { of squares }\end{array}$ & gl & $\begin{array}{c}\text { Root mean } \\
\text { square }\end{array}$ & F & Sig. \\
\hline $\begin{array}{l}\text { Corrected } \\
\text { Model }\end{array}$ & $8963.716 a$ & 9 & 995.968 & 23.737 & .000 \\
\hline Intersection & 394.702 .910 & 1 & 394.702 .910 & 9.407 .017 & .000 \\
\hline Resin & 5.007 & 1 & 5.007 & .119 & .731 \\
\hline $\begin{array}{l}\text { Polished } \\
\text { Group }\end{array}$ & 8.241 .828 & 4 & 2.060 .457 & 49.107 & .000 \\
\hline $\begin{array}{l}\text { Resin * } \\
\text { Polished } \\
\text { Group }\end{array}$ & 716.882 & 4 & 179.220 & 4.271 & .004 \\
\hline Error & 2.937 .085 & 70 & 41.958 & & \\
\hline Total & 406.603 .711 & 80 & & & \\
\hline $\begin{array}{l}\text { Corrected } \\
\text { Total }\end{array}$ & 11.900 .801 & 79 & & & \\
\hline
\end{tabular}

r2 $=.753$ (adjusted r2 =.721)

The control group (Mylar strips) obtained the highest gloss values, followed by group G5 (ENA Shiny) without significant differences, G3 (Composite Politur), and G4 and G2 without significant differences. Since the interaction was found to be significant $(p=0.004)$, there were Resin-Polishing system combinations where the effect of each of the factors affects potency gloss values

Representative SEM images of each resin after the finishing and polishing procedure and related polishing system are shown in Figures 1 to 4 .

Clearly smooth surfaces resulted in control groups for both resins. For Filtek Bulk-Fill Posterior, some voids can be noted in the control group according to Figure 1A. Smooth surfaces can also be noted for both resins polished with the ENA Shiny system (3-step polishing pastes). The most irregular surface with voids and the appearance of filler detachment was obtained for both bulk-fill resins polished with aluminum oxide discs (SofLex XT, Figures 1C and 2C) and Soflex Spiral Wheels (1D and 2D), followed by Composite Politur system used with Tetric N Bulk-Fill (Figure 2D). Figure 3 shows $\times 3500$ magnification of the polished surfaces of Filtek BulkFill Posterior. Tetric N Bulk-Fill showed a rougher surface with voids and filler content detachment at x3500 magnification (Figure 4).

\section{DISCUSSION}

The first null hypothesis, "there are no significant differences in gloss values between the different polishing systems of the bulk-fill composite 
Table 3:

\begin{tabular}{l|c|c|c|c|c}
\hline & $\begin{array}{l}\text { Control } \\
\text { Group }\end{array}$ & $\begin{array}{c}\text { SofLex } \\
\text { XT }\end{array}$ & $\begin{array}{c}\text { Composite } \\
\text { Politur }\end{array}$ & $\begin{array}{c}\text { Soflex } \\
\text { Spiral }\end{array}$ & $\begin{array}{c}\text { ENA } \\
\text { Shiny }\end{array}$ \\
\hline $\begin{array}{l}\text { Control } \\
\text { Group }\end{array}$ & - & $* *$ & N.S. & $* *$ & N.S. \\
\hline SofLex XT & $* *$ & - & $* *$ & N.S. & $* *$ \\
\hline $\begin{array}{l}\text { Composite } \\
\text { Politur }\end{array}$ & N.S. & $* *$ & - & $* *$ & N.S. \\
\hline Soflex Spiral & $* *$ & N.S. & $* *$ & - & $* *$ \\
\hline \multicolumn{7}{l}{ ENA Shiny } & N.S. & $* *$ & N.S. & $* *$ & - \\
\hline
\end{tabular}

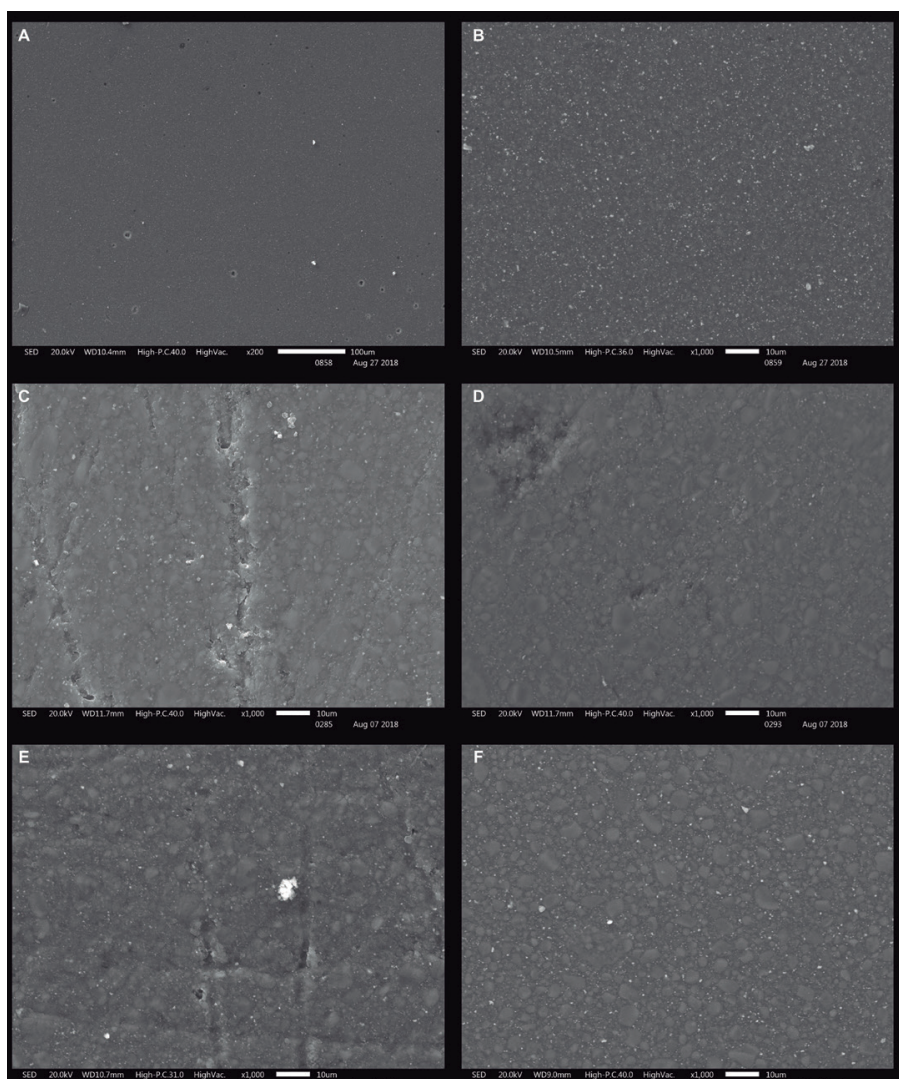

Figure 1. Filtek BulkFill Posterior SEM. A and B: Control group (Mylar Strips, No polishing) at $x 200$ and $x 1000$,respectively. C: $x 1000$ SEM SofLex XT. D: x1000 Composite Politur. D:x1000 Soflex Spiral, and E: ENA Shiny.

resin tested" was rejected. Significant statistical differences were found between each one system used in the study ( $p$ value $=0.000$ ). The second null hypotheses, "there are no significant differences in gloss values between resins for the same polishing system", was accepted. No differences between bulk-fill composite resins were statistically significant when the resin was polished with the same system ( $p$ value $=0.73$ ). This finding confirms that a careful selection of polishing systems is crucial in order to obtain high gloss values for bulk-fill resin composites.

Surface gloss values (measured in $\mathrm{GU}$ ) of natural teeth have been previously established by the American Dental Association (ADA), which is considered to be between 40 to $60 \mathrm{GU}^{(19)}$. The control group of this study, represented by the polymerization through the use of mylar strips and without polishing and finishing technique, represented the highest gloss values (mean gloss value $80.7 \pm 7.7 \mathrm{GU}$ ). According to Hachiya et al. ${ }^{(20)}$, this kind of finished surface is not adequate, because a surface richer in polymer may be obtained, which would be highly susceptible to void formation and composite resin discoloration, and not recommended as a properly finished polished surface. Similar results were obtained with polishing pastes such as the ENA Shiny system (mean value: 79.6 $\pm 5.7 \mathrm{GU}$ ), and for Filtek BulkFill Posterior, which was the bulk-fill composite resin with the highest gloss value (83.6 $4.8 \mathrm{GU})$. Comparing this result with conventional composite resins, several studies ${ }^{(21)}$, including a thorough systematic review from Kaizer et al. ${ }^{(22)}$, proved that nanofilled composite resins containing "nanoclusters" obtained

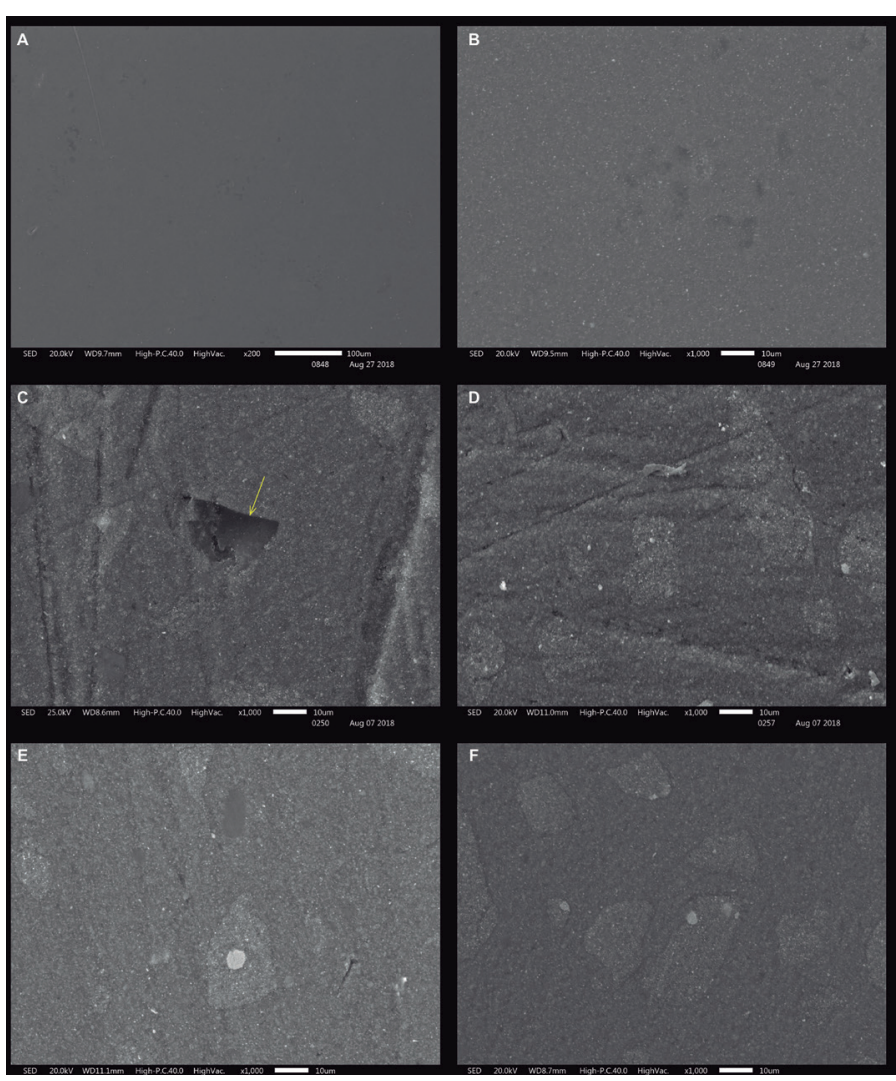

Figure 2. Tetric N Bulk-Fill SEM. A and B: Control group (Mylar Strips, No polishing) at x200 and x1000,respectively. C: x1000 SEM SofLex XT (yellow arrow, surface voids). D: x1000 Composite Politur. D:x1000 Soflex Spiral, and E: ENA Shiny.

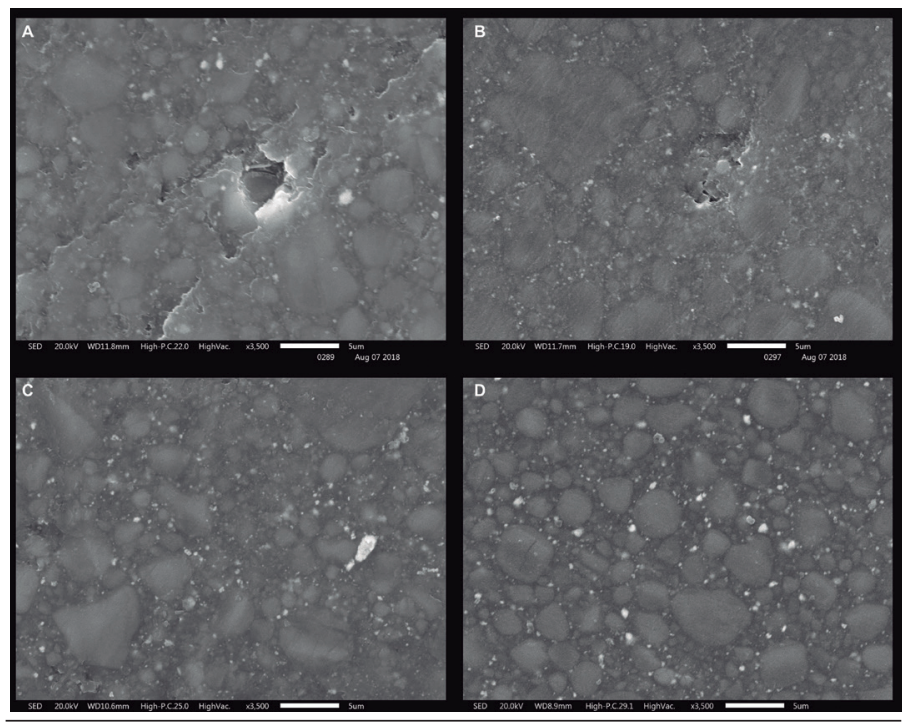

Figure 3. Filtek BulkFill Posterior SEM. X3500 magnification. A: Clear void for Filtek BulkFill Posterior surface polished with SofLex XT. B: Composite Politur void formation. C: Soflex Spiral detachment particles in the composite resin surface, and D: regular surface polished with ENA Shiny.

the highest gloss values. For this case, Filtek Bulk-Fill Posterior has the same particle technology coming from Filtek Z350 XT or Filtek Supreme from the same manufacturer (3M ESPE), even though bulk-fill composite resins have lower gloss values than conventional composite resins. In terms of SEM and GU values, Filtek Bulk-Fill Posterior Restorative has the smoothest surface (polished with ENA Shiny polishing system), which is also comparable to conventional nanofilled composite resins from the same company when it comes to the evaluation of surface roughness ${ }^{(23)}$.

The lowest gloss values were obtained with impregnated aluminum oxide discs (SofLex XT) with mean values of $57.8 \pm 5.8 \mathrm{GU}$. Similar results were obtained with Soflex Spiral Wheels: $58.4 \pm 8.7$. While they have been shown 
to be inferior to the other polishing systems in this study, they're still within the range of natural teeth according to the ADA. Even so, the SEM images showed voids and filler particle detachment, which may increase surface roughness and reduce gloss with results that are potentially detrimental for surface maintenance (x3500 SEM magnification, Figures 3 and 4). Tetric $N$ Ceram demonstrated irregular behavior in terms of void formation and filler particle detachment for every polishing system according to figure 4 , which may influence bacteria colonization and modification of surface gloss over time.

During a study performed by O'Neill et al. ${ }^{(24)}$, four high viscosity bulk-fill resins and one flowable bulk-fill composite resin were evaluated under tooth brushing after 5000, 10000 and 15000 brushing cycles. Of the studied bulkfill composite resins, after 5000 brushing cycles, only Filtek One Bulk Fill and SDR flow maintained acceptable gloss retention $(68.7 \pm 5.1 \mathrm{GU}$ and $48.9 \pm$ $14.0 \mathrm{GU}$, respectively). After 15000 brushing cycles, only Filtek One Bulk Fill maintained gloss $(43.8 \pm 4.8 \mathrm{GU})$, which is still acceptable and considered to

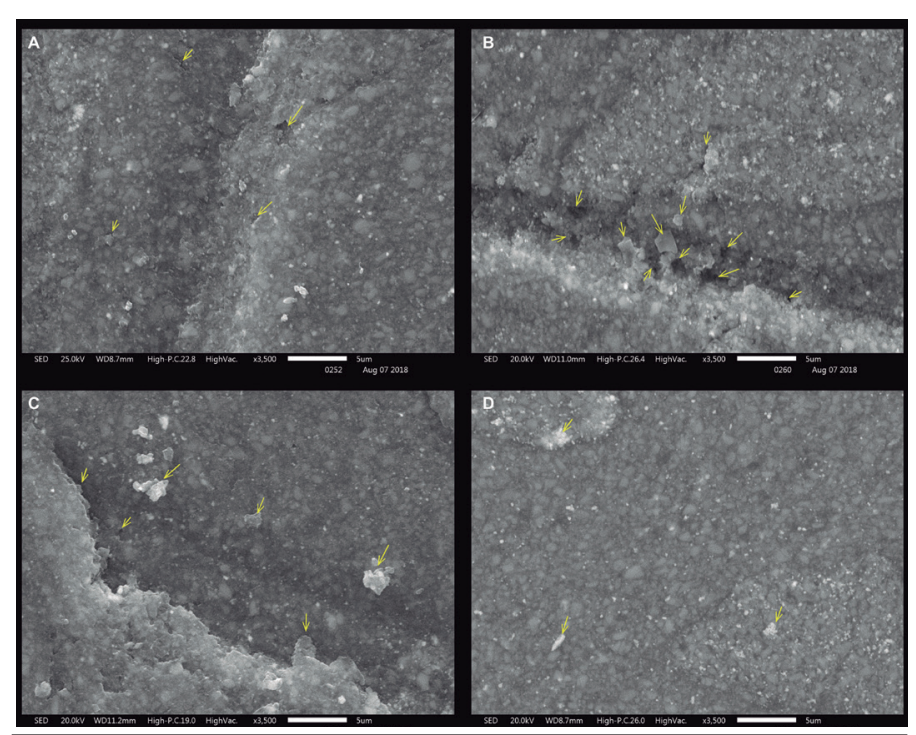

Figure 4. Tetric N Bulk-Fill SEM- $x 3500$ magnification. A: yellow arrows show voids in the surface polished with SofLex XT. B: yellow arrows show free filler particles and voids when the surface is polished with Composite Politur. C: yellow arrows show particle detachment and voids for a Soflex Spiral polished surface, and D: the most regular surface is shown with ENA Shiny system, although some free particles may be noted. be similar to natural teeth according to the ADA. Meanwhile, SonicFill2, Tetric Evoceram Bulk Fill, SDR flow and Admira Fusion X-tra lost gloss considerably $(10.0 \pm 2.6 \mathrm{GU}, 14.1 \pm 3.8 \mathrm{GU}, 11.9 \pm 4.1 \mathrm{GU}$, and $2.8 \pm 0.3 \mathrm{GU}$, respectively) after 15000 brushing cycles, also increasing surface roughness. This study showed that bulk-fill composites are rougher than conventional composite resins and that there is an inverse linear relationship between surface gloss and surface roughness. This would suggest that tooth brushing increases roughness and reduces gloss, increasing the possibility of biofilm retention which may be detrimental for the bonding tooth-restoration interphase. This is the only published article found where gloss surface of bulk-fill composite resins were evaluated. On the contrary, a study from Rigo et al. ${ }^{(25)}$ concluded that roughness is not influenced by the polishing system applied over nanohybrid bulk-fill compactable composite resins such as Tetric EvoCeram Bulk-Fill. These authors used SofLex XT aluminum oxide discs and Astropol rubber points, and concluded that SofLex XT produced a rougher surface for every bulk-fill composite tested. In the present study, similar results were found according to the SEM images. This is the only comparable variable from the Rigo et al(25). study and ours, because Astropol is a three step rubber point, and Composite Politur are two-step diamond points. Even so, their study confirms the SEM results found in the present study.

Although there are few studies available for comparing the results obtained in relation to the gloss surface of these two bulk-fill composite resins, it must be noted that the gloss surface measurement was performed immediately after the polishing technique, so studies that evaluate the optical behavior of these resins over time are still necessary. Also, the findings showed gloss mean values that are within the gloss natural values recommended from the ADA (40-60 GU)

\section{CONCLUSIONS}

Within the limitations of the present study, the authors conclude that the polishing systems that were tested obtained acceptable in vitro gloss results for both bulk-fill composite resins. Diamond paste is the system with the best performance, followed by Diamond rubber points. Aluminum oxide impregnated discs, as well as thermoplastic elastomer wheels impregnated with aluminum oxide particles had the lowest gloss behavior, although all systems have acceptable gloss values according to ADA recommendations. SEM images showed an irregular surface for each resin polished with aluminum oxide systems. The authors recommend future studies that include surface gloss and roughness over time.

\section{ACKNOWLEDGEMENT}

The authors want to express their acknowledgement to Prof. Luis Barrios, who kindly did the statistics of the present article. Also we personally thank the Internal Funding source Code VRIIP0025-16 of the Vice Rectory of Research, Innovation and Postgraduate of Universidad Arturo Prat, lquique Chile.

\section{References}

1.- Devji T. Direct light-activated resin-based composite restorations in posterior teeth seem to have a high clinical success rate and long-term survival. J Am Dent Assoc. 2017; 148(10):e160.

2.- Ferracane J. Resin composite-State of the art. Dent Mater. 2011;27(1):29-38.

3.- Kaisarly D, Gezawi M. Polymerization shrinkage assessment of dental resin composites: a literature review. Odontology. 2016;104(3):257-270.

4.- Chi H. A Posterior composite case utilizing the incremental and stratified layering technique. Oper Dent. 2006;31(4):512-516

5.- Durán Ojeda G, Henríquez Gutiérrez I, Tisi J, Báez Rosales A. A novel technique for bulk-fill resin-based restorations: achieving function and esthetics in posterior teeth. Case Rep Dent. 2017;2017:1-5.

6.- Bouillaguet S, Gamba J, Forchelet J, Krejci I, Wataha J. Dynamics of composite polymerization mediates the development of cuspal strain. Dent Mater. 2006;22(10):896-902 7.- Van Ende A, De Munck J, Lise DP, Van Meerbeek B. Bulk-Fill Composites: A review of the current literature. J Adhes Dent. 2017;19(2):95-109.

8.- Hirata R, Kabbach W, de Andrade O, Bonfante E, Giannini M, Coelho P. Bulk Fill Composites: An anatomic sculpting technique. J Esthet Restor Dent. 2015;27(6):335-343. 9.- Ilie N, Bucuta S, Draenert M. Bulk-fill resin-based composites: an in vitro assessment of their mechanical performance. Oper Dent. 2013;38(6):618-625.

10.- Leprince J, Palin W, Vanacker J, Sabbagh J, Devaux J, Leloup G. Physicomechanical characteristics of commercially available bulk-fill composites. J Dent. 2014;42(8):993-1000.

11.- Alshali R, Silikas N, Satterthwaite J. Degree of conversion of bulk-fill compared to conventional resin-composites at two time intervals. Dent Mater. 2013;29(9):e213-e217. 12.- Li X, Pongprueksa P, Van Meerbeek B, De Munck J. Curing profile of bulk-fill resinbased composites. J Dent. 2015;43(6):664-672

13.- Heck K, Manhart J, Hickel R, Diegritz C. Clinical evaluation of the bulk fill composite QuiXfil in molar class I and II cavities: 10-year results of a RCT. Dent Mater. 2018;34(6):e138-e147.

14.- Garoushi S, Vallittu P, Shinya A, Lassila L. Influence of increment thickness on light transmission, degree of conversion and micro hardness of bulk fill composites.
Odontology. 2015;104(3):291-297

15.- Shamszadeh S, Sheikh-Al-Eslamian SM, Hasani E, Abrandabadi AN, Panahandeh $\mathrm{N}$. Color stability of the bulk-fill composite resins with different thickness in response to coffee/water inmersion. International J Dent. 2016; 2016:7186140.

16.- Erdemir U, Kaner A, Eren M, Ozan G, Yıldız E. Color stability of bulk-fill composites immersed in different drinks. Color Res Appl. 2018;43(5):785-793.

17.- Lefever D, Perakis N, Roig M, Krejci I, Ardu S. The effect of toothbrushing on Surface gloss in resin composites. Am J Dent. 2012;25(1):54-58.

18.- Antonson S, Yazici A, Kilinc E, Antonson D, Hardigan P. Comparison of different finishing/polishing systems on surface roughness and gloss of resin composites. J Dent. 2011;39:e9-e17.

19.- Kamonkhantikul K, Arksornnukit M, Takahashi H, Kanehira M, Finger W. Polishing and toothbrushing alters the surface roughness and gloss of composite resins. Dent Mater J. 2014;33(5):599-606.

20.- Hachiya $\mathrm{Y}$, Iwaku M, Hosoda $\mathrm{H}$, Fusuyama T. Relation of finish to discoloration of composite resins. J Prosth Dent. 1984;52:811-814.

21.- Rodrigues-Junior S, Chemin P, Piaia P, Ferracane J. Surface roughness and gloss of actual composites as polished with different polishing systems. Oper Dent. 2015;40(4):418-429.

22.- Kaizer M, de Oliveira-Ogliari A, Cenci M, Opdam N, Moraes R. Do nanofill or submicron composites show improved smoothness and gloss? A systematic review of in vitro studies. Dent Mater. 2014;30(4):e41-e78.

23.- Senawongse $P$, Pongprueska $P$. Surface roughness of nanofill and nanohybrid resin composites after polishing and brushing. J Esthet Restor Dent. 2007:19(5):265-273.

24.- O'Neill C, Kreplak L, Rueggeberg F, Labrie D, Shimokawa C, Price R. Effect of tooth brushing on gloss retention and surface roughness of five bulk-fill resin composites. $J$ Esthet Restor Dent. 2017;30(1):59-69.

25.- Rigo L, Bordin D, Fardin V, Coelho P, Bromage T, Reis A et al. Influence of polishing system on the surface roughness of flowable and regular-viscosity bulk fill composites. Int J Periodontics Restorative Dent. 2018;38(4):e79-e86. 